\title{
Dielectric Study of Dynamical Heterogeneity in Blends of Polystyrene and Low Mass Compounds
}

\author{
Hironobu HoRI, Osamu URAKAWA, and Keiichiro ADACHI ${ }^{\dagger}$ \\ Department of Macromolecular Science, Graduate School of Science, Osaka University, \\ Toyonaka, Osaka 560-0043, Japan
}

(Received May 12, 2003; Accepted June 25, 2003)

\begin{abstract}
We investigated the dielectric relaxation in blends of 4-n-pentyl-4'-cyanobiphenyl (5CB) and polystyrene (PS) in order to examine the size effect of low mass molecules on the cooperativity of local dynamics. To obtain the dielectric data in the isotropic state, the phase diagram was produced. The temperature $(T)$ dependence of the dielectric loss factor $\varepsilon^{\prime \prime}$ of PS/5CB blends containing 20 to $30 \mathrm{wt} \%$ of $5 \mathrm{CB}$ exhibited a bimodal peak near the calorimetric glass transition temperature $T_{\mathrm{g}}$. The bimodal peak was resolved into two peaks termed $\alpha$ and $\beta$. The $\alpha$ process locates above $T_{\mathrm{g}}$ and can be assigned to cooperative motions of the PS segments and the 5CB molecules. The $\beta$ process is responsible to independent reorientation of the 5CB molecules in the glassy PS matrix. The $\alpha$ and $\beta$ loss peaks tend to merge with increasing $T$. This behavior contrasts to the dielectric behavior of the PS/toluene system which exhibits loss peaks due to segmental motions of PS and rotation of the toluene molecules in well separated temperature regions. The toluene molecule has a size of $c a .1 / 3$ of the 5CB molecule and does not move cooperatively with PS but the 5CB molecule does. From the intensity of the $\alpha$ and $\beta$ peaks, the fraction of the 5CB molecules moving cooperatively with PS has been estimated to be 0.25 and 0.63 for the blends containing 30 and $20 \mathrm{wt} \%$ of $5 \mathrm{CB}$, respectively.

KEY WORDS Polystyrene / 4- $n$-Pentyl-4'-cyanobiphenyl / Dielectric Relaxation / Dynamical Heterogeneity / Cooperativity / Diluent / Segmental Motion /
\end{abstract}

Dynamics of low molecular weight molecules dissolved in polymer matrices has been studied long by means of dielectric, ${ }^{1-8}$ mechanical, ${ }^{7,8} \mathrm{NMR},{ }^{6,7,9,10}$ light scattering, ${ }^{7}$ fluorescence depolarization, ${ }^{11} \mathrm{ESR},{ }^{12}$ and thermal methods. ${ }^{6,13}$ Adachi et al. ${ }^{4}$ and Yoshizaki et al. ${ }^{1}$ reported the dielectric behaviors of the systems consisting of polystyrene (PS) and alkylbenzene such as toluene, ethylbenzene and butylbenzene. It was reported that the relaxation due to rotation of the solvent molecules ( $\beta$ process) and that due to segmental motions of PS ( $\alpha$ process) occur separately in different temperature-frequency regions. ${ }^{1,4}$ Similar bimodal mobilities of components are reported for various systems consisting of large and small molecules. ${ }^{5,6,9,10}$ This behavior is not in harmony with a generally believed view of cooperativity ${ }^{14-18}$ of local motions in supercooled liquids near the glass transition temperature $T_{\mathrm{g}}$. For instance if the PS chains and the toluene molecules in the PS/toluene system move cooperatively, the splitting into the two different relaxation processes would not occur. Such behaviors have been reported even for miscible polymer blends and are called "dynamical heterogeneity". ${ }^{19,}{ }^{20}$ However in those literatures referred above, the interest has been centered mostly on the spectral shapes of the relaxation processes and the relationship between the dynamical heterogeneity and the cooperativity has been scarcely investigated.

What factors result in the dynamical heterogeneity in polymer/diluent systems? An intuitive conjecture to this question is that if the size of the dynamical units of the polymer segments is much larger than the size of the diluent molecule, their motions are decoupled. Conversely if a diluent with a large size is mixed in a polymer, the diluent molecules are expected to move cooperatively with the polymer segments. The other factor which must be taken into account is intermolecular interactions. However this factor is considered to be a minor factor as far as mixtures of organic compounds are concerned, since various polymer/diluent systems exhibit similar behaviors of the dynamical heterogeneity. Therefore in this paper, we focus our attention on the size effect of diluents and have examined the dielectric behavior of blends of PS and 4-n-pentyl-4' -cyanobiphenyl (5CB) whose structure is given by $\mathrm{C}_{5} \mathrm{H}_{11}-\mathrm{C}_{6} \mathrm{H}_{4}-\mathrm{C}_{6} \mathrm{H}_{4}-\mathrm{CN}$. The $5 \mathrm{CB}$ molecule is $c a$. three times larger than the toluene molecule and the dielectric behaviors of PS/5CB and PS/toluene systems have been compared. It is known that $5 \mathrm{CB}$ is a liquid crystalline compound and $5 \mathrm{CB} / \mathrm{PS}$ blends are expected to exhibit complicated phase behavior as observed in similar blends. ${ }^{22-25}$ Since our interest is in the behavior in the isotropic mixture of 5CB and PS, we have to make sure that measurements are made on the isotropic blend. For this reason, the phase behavior of the $\mathrm{PS} / 5 \mathrm{CB}$ is reported briefly and then the dielectric behavior of $\mathrm{PS} / 5 \mathrm{CB}$ blends is reported in detail.

${ }^{\dagger}$ To whom correspondence should be addressed (Phone/Fax: 06-6850-5464, E-mail: adachi@chem.sci.osaka-u.ac.jp). 


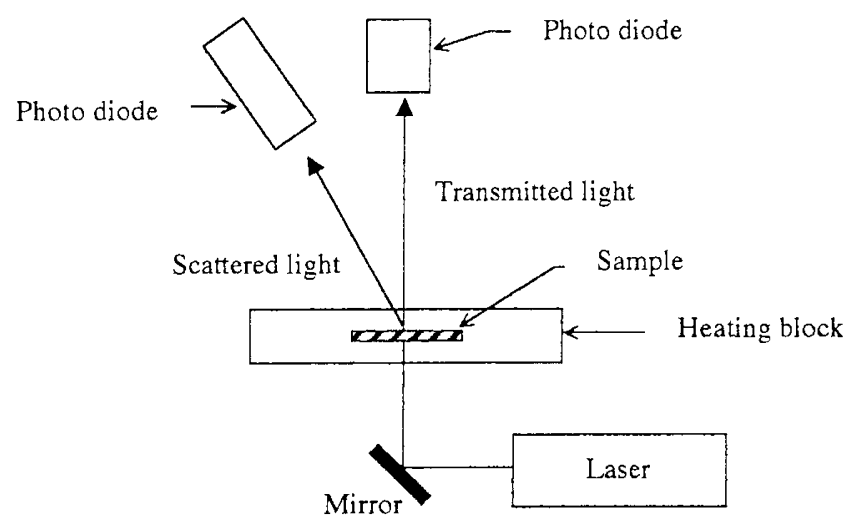

Figure 1. Schematic illustration of an apparatus for observation of turbidity of blend samples.

\section{EXPERIMENTAL}

\section{Materials}

Polystyrene was prepared by anionic polymerization with sec-butyllithium as the initiator. The molecular weight and the $M_{\mathrm{w}} / M_{\mathrm{n}}$ ratio were determined to be 57800 and 1.03, respectively, by comparing the gel permeation chromatograph (GPC) diagrams of the PS and standard polystyrenes purchased from Tosoh (Tokyo, Japan). The sample of 5CB was purchased from Merck-Japan (Tokyo) and used without further purification. Blends of PS/5CB were prepared by dissolving prescribed amounts of PS and 5CB in methylethylketone and then casting the solutions into film in an ambient atmosphere. The cast films were further dried in vacuum of $0.01 \mathrm{~Pa}$ at $350 \mathrm{~K}$ for several days. Dielectric measurements were carried out on three blends coded as $\mathrm{PS} / 5 \mathrm{CB}(7 / 3), \mathrm{PS} / 5 \mathrm{CB}(8 / 2)$, and $\mathrm{PS} / 5 \mathrm{CB}(9 / 1)$ in which the content of $5 \mathrm{CB}$ is 31,21 , and $8 \mathrm{wt} \%$, respectively.

\section{Methods}

The phase behavior was investigated by using a polarizing microscope (Nikon E-400, Tokyo) and a homemade light-scattering apparatus as shown schematically in Figure 1. Dielectric measurements were carried out with an RLC bridge (QuadTech model 7600, Maynard, USA) over frequency range from $10 \mathrm{~Hz}$ to $2 \mathrm{MHz}$ and temperature range from 100 to $360 \mathrm{~K}$. Measurements of differential scanning calorimetry (DSC) were made at the heating rate of $10 \mathrm{~K} \mathrm{~min}^{-1}$ with an apparatus (Seiko SSC-580, Tokyo).

\section{RESULTS AND DISCUSSION}

\section{Phase Diagram}

Figure 2 shows the phase diagram of the PS/5CB

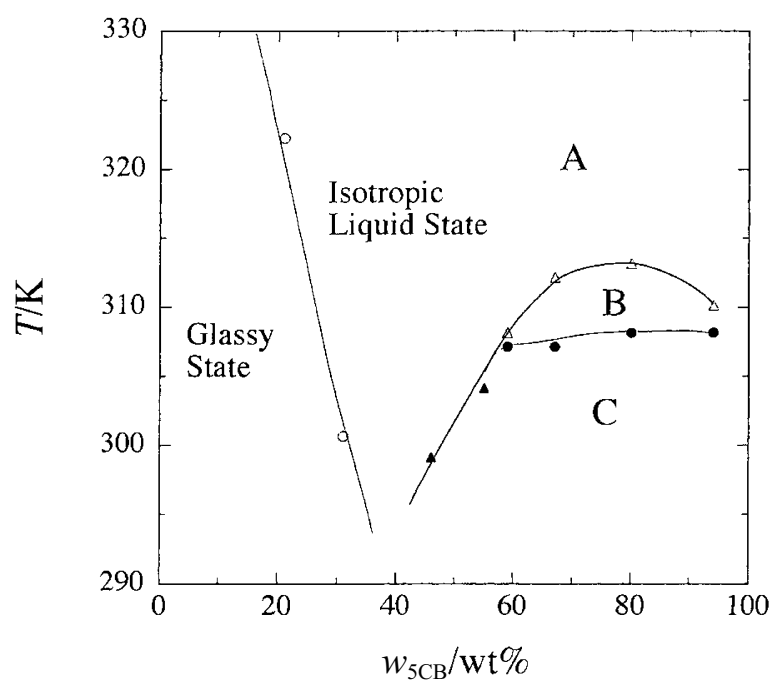

Figure 2. Phase diagram of the $\mathrm{PS} / 5 \mathrm{CB}$ system. The phases $\mathrm{A}$ and $\mathrm{B}$ are the isotropic one phase and isotropic two phase regions, respectively. In the region $\mathrm{C}$ the isotropic $\mathrm{PS}$ rich solution and liquid crystalline $5 \mathrm{CB}$ coexist. The open circle indicates $T_{\mathrm{g}}$ in the isotropic solution state.

system determined by measurements of light scattering and by observation with a polarizing microscope. There are three regions $\mathrm{A}, \mathrm{B}$, and $\mathrm{C}$ as reported for systems consisting of a polymer and an alkylcyanobiphenyl. ${ }^{22-25}$ The region $\mathrm{A}$ is the isotropic solution state. Since 5CB is a poor solvent for PS, the liquidliquid phase separation occurs at the boundary between $\mathrm{A}$ and $\mathrm{B}$. In the region $\mathrm{B}$, the isotropic $5 \mathrm{CB}$ rich phase and the PS rich phases coexist. In the region $\mathrm{C}$, the isotropic PS rich phase and liquid crystalline 5CB (nematic) coexist. The open circles indicate $T_{\mathrm{g}}$ in the isotropic state. We see that $T_{\mathrm{g}}$ decreases steeply with increasing $5 \mathrm{CB}$ content indicating that $5 \mathrm{CB}$ acts as a plasticizer. Dielectric measurements were performed in the isotropic state.

\section{Dielectric Behavior}

Figures 3 and 4 show the temperature $(T)$ dependencies of the dielectric loss factor $\varepsilon^{\prime \prime}$ for $\mathrm{PS} / 5 \mathrm{CB}(7 / 3)$ and $\mathrm{PS} / 5 \mathrm{CB}(8 / 2)$, respectively. It is recognized that the $\varepsilon^{\prime \prime}$ curves at 50 and $100 \mathrm{~Hz}$ consist of two loss peaks and those two peaks tend to merge at high frequencies. Figures $5 \mathrm{a}$ and $5 \mathrm{~b}$ show the frequency dependencies of $\varepsilon^{\prime \prime}$ for $\mathrm{PS} / 5 \mathrm{CB}(7 / 3)$ and $\mathrm{PS} / 5 \mathrm{CB}(8 / 2)$, respectively. Corresponding to the $T$-dependence curves (Figures 3 and 4), the frequency dependence curves are also bimodal in temperature region from 300 to $310 \mathrm{~K}$. We term the low and high frequency modes $\alpha$ and $\beta$, respectively. Obviously the high and low temperature modes observed in Figures 3 and 4 correspond to the $\alpha$ and $\beta$ processes, respectively. 


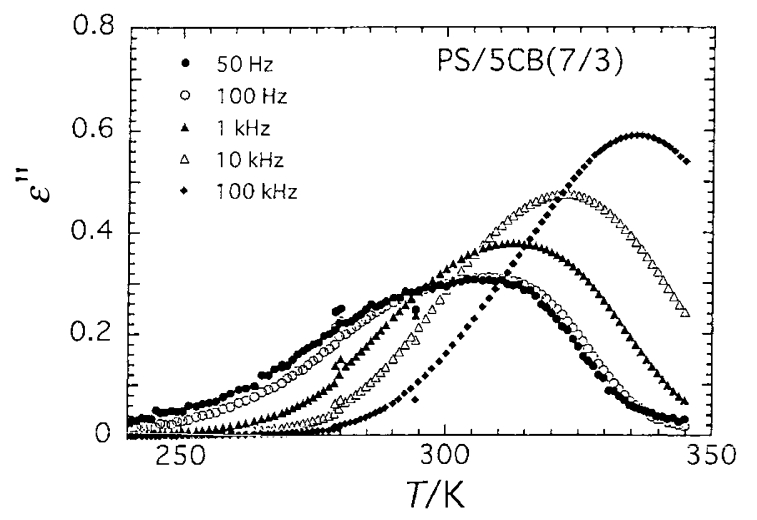

Figure 3. Temperature dependence of dielectric loss factor $\varepsilon^{\prime \prime}$ for $\mathrm{PS} / 5 \mathrm{CB}(7 / 3)$.

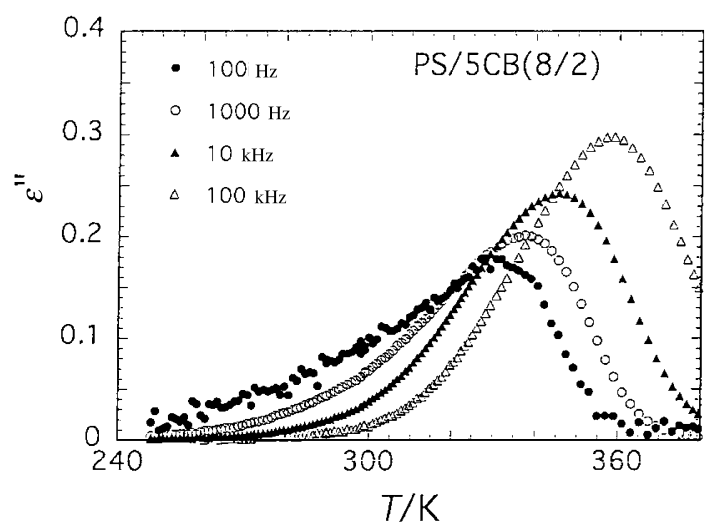

Figure 4. Temperature dependence of dielectric loss factor $\varepsilon^{\prime \prime}$ for $\mathrm{PS} / 5 \mathrm{CB}(8 / 2)$.

\section{Analysis of Dielectric Data}

In order to examine the behavior of the $\alpha$ and $\beta$ processes, we attempted to resolve the observed $\varepsilon^{\prime \prime}$ curves into the $\alpha$ and $\beta$ peaks. We could not resolve the frequency dependence of $\varepsilon^{\prime \prime}$ due to limited frequency range in the low frequency side and therefore we resolved the $T$-dependence curves. We used an empirical equation given by eq 1 assuming that each peak has a symmetrical shape against $T$ for the sake of simplicity:

$$
\varepsilon^{\prime \prime}(T)=\sum_{\mathrm{j}} \frac{K_{\mathrm{j}}}{\mathrm{e}^{\gamma_{\mathrm{j}}\left(T-T_{\mathrm{j}}\right)}+\mathrm{e}^{-\gamma_{\mathrm{j}}\left(T-T_{\mathrm{j}}\right)}}
$$

where $K_{\mathrm{j}}, \gamma_{\mathrm{j}}$, and $T_{\mathrm{j}}$ are the constants and $\mathrm{j}$ represents $\alpha$ and $\beta$. The reason for use of eq 1 is as follows. The Debye theory predicts that $\varepsilon^{\prime \prime} v s . \log \omega$ is given by the hyperboric secant function $\operatorname{sech}(x)$ where $\omega$ is the angular frequency and $x=\log \omega$. Since the Debye function is a function of $\omega \tau$, the $\varepsilon^{\prime \prime} v s . \log \tau$ is also given by the same form where $\tau$ is the relaxation time. In the relatively narrow temperature range where the $\varepsilon^{\prime \prime}$ peak appears in the $\varepsilon^{\prime \prime} v s$. $T$ curve, $\log \tau$ is proportional to $1 / T$ since $\tau$ is given by the Arrhenius equation. We further approximate that $1 / T$ is proportional to $-T$. Then the observed $T$ dependence curves are expected to be
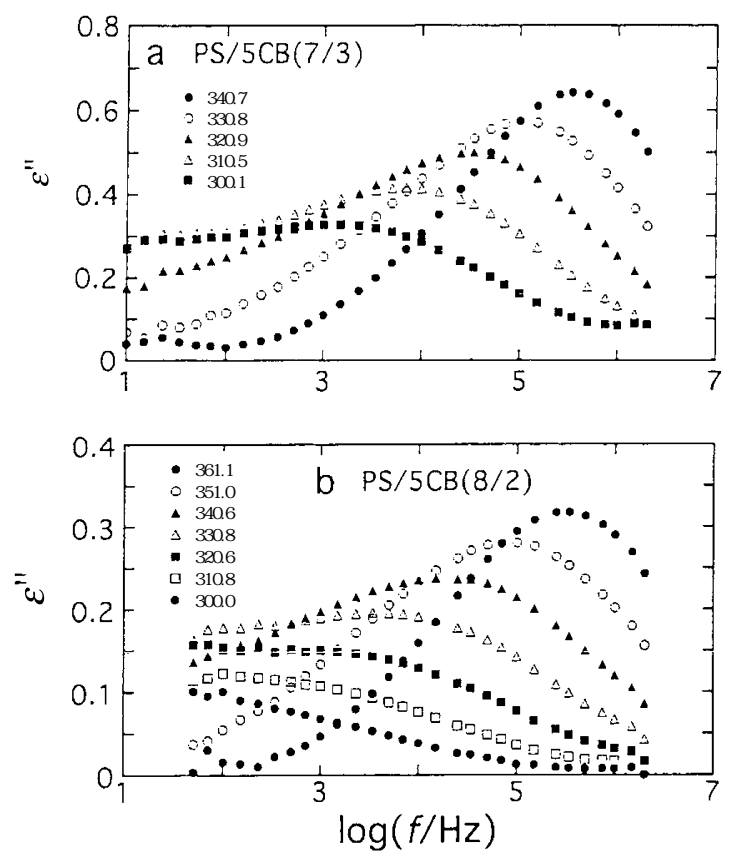

Figure 5. Frequency dependencies of $\varepsilon^{\prime \prime}$ for $\operatorname{PS} / 5 \mathrm{CB}(7 / 3)$ (a) and for $\mathrm{PS} / 5 \mathrm{CB}(8 / 2)(\mathrm{b})$.

represented by a form of eq 1 with the important parameter $\gamma$ representing the broadness of the loss curve. Irrespective of the theoretical background, we use eq 1 as an empirical equation and therefore if it does not work, we will seek new equations.

The examples of the resolved peaks are shown in Figure 6 . We see that eq 1 works well to reproduce the experimental $\varepsilon^{\prime \prime}$. However we see that the agreement is relatively poor only in the high temperature region. Probably this is because the $\alpha$ process has unsymmetrical spectra. ${ }^{26}$ The values of the parameters appearing in eq 1 are listed in Table I.

The Arrhenius plots for the $\alpha$ and $\beta$ loss peaks thus resolved are shown in Figure 7, in which the frequency $\mathrm{f}$ used for measurements of the $T$-dependence of $\varepsilon^{\prime \prime}$ is plotted against the inverse of the temperature $T_{\max }$ where the resolved $\varepsilon^{\prime \prime}$ curves exhibit maxima. It is seen that the $\alpha$ and $\beta$ loss peaks tend to merge with increasing $T$ and that the behavior is very similar to the primary and secondary relaxations of amorphous polymers. ${ }^{26}$ From the slope of the Arrhenius plot, the apparent activation energy $E$ was determined and listed in Table II. It is well known that the Arrhenius plot for the $\alpha$ process conforms to the Vogel-Fulcher equation. ${ }^{27,28}$ The present value of $E$ corresponds to the average activation energy in the frequency range from $100 \mathrm{~Hz}$ to $1 \mathrm{kHz}$.

\section{Assignment of the $\alpha$ and $\beta$ Processes}

Figure 8 shows the temperature dependence curves of $\log \varepsilon^{\prime \prime}$ at $100 \mathrm{~Hz}$ for $\mathrm{PS} / 5 \mathrm{CB}(7 / 3)$ and $\mathrm{PS} /$ toluene 
Table I. Values of parameters of eq 1

\begin{tabular}{crcccccccc}
\hline code & $f / \mathrm{Hz}$ & $K_{\alpha}$ & $\gamma_{\alpha}$ & $T_{\alpha} / \mathrm{K}$ & $\Delta \varepsilon_{\alpha}$ & $K_{\beta}$ & $\gamma_{\beta}$ & $T_{\beta} / \mathrm{K}$ & $\Delta \varepsilon_{\beta}$ \\
\hline$(7 / 3)$ & 23 & 0.30 & 0.12 & 312 & 0.91 & 0.50 & 0.047 & 289 & 2.30 \\
& 50 & 0.25 & 0.13 & 314 & 0.69 & 0.51 & 0.056 & 292 & 1.93 \\
& 100 & 0.24 & 0.13 & 317 & 0.65 & 0.56 & 0.055 & 298 & 2.07 \\
& 230 & 0.24 & 0.13 & 319 & 0.64 & 0.58 & 0.058 & 301 & 1.99 \\
& 500 & 0.24 & 0.13 & 321 & 0.63 & 0.62 & 0.061 & 304 & 1.98 \\
& 1000 & 0.21 & 0.13 & 325 & 0.54 & 0.70 & 0.064 & 308 & 2.08 \\
$(8 / 2)$ & 50 & 0.27 & 0.105 & 331 & 0.90 & 0.20 & 0.041 & 300 & 0.63 \\
& 100 & 0.26 & 0.102 & 332 & 0.89 & 0.19 & 0.044 & 306 & 0.53 \\
& 230 & 0.28 & 0.096 & 334 & 1.01 & 0.18 & 0.048 & 309 & 0.45 \\
& 500 & 0.20 & 0.092 & 338 & 0.73 & 0.20 & 0.050 & 322 & 0.44 \\
& 1000 & 0.21 & 0.092 & 339 & 0.76 & 0.20 & 0.050 & 324 & 0.44 \\
\hline
\end{tabular}
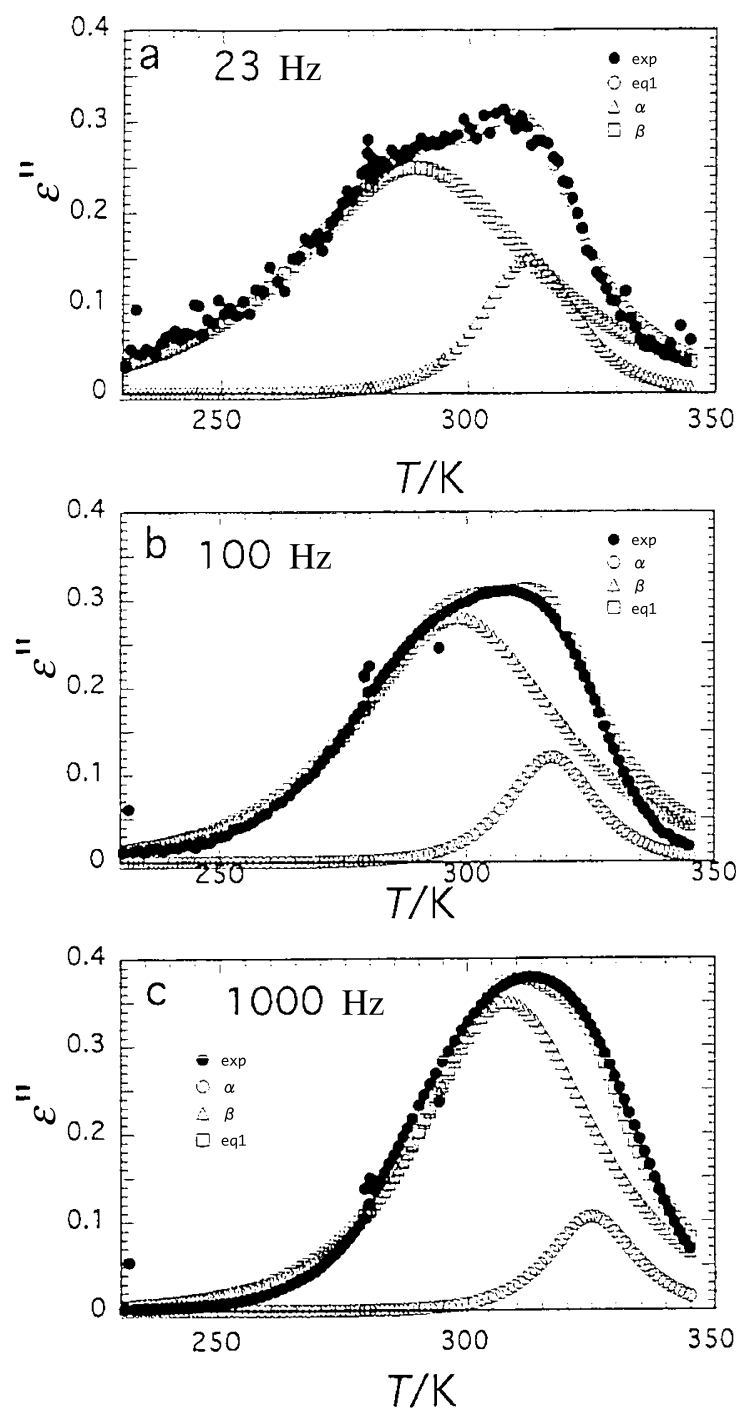

Figure 6. Examples of the curve fitting of the experimental $\varepsilon^{\prime \prime}$ curve (closed circle) to eq 1 (open circle). The plots with triangle and square keys indicate the resolved $\alpha$ and $\beta$ peaks, respectively.

(7/3) reported by Yoshizaki et al. ${ }^{1}$ As is seen in this figure the PS/toluene blend exhibits a loss curve well separated into the $\alpha$ and $\beta$ peaks which can be assigned to segmental motions of PS and rotation of the toluene molecules, respectively, since the intensity of the $\alpha$ peak increases with increasing PS content. ${ }^{1,4}$ This as-
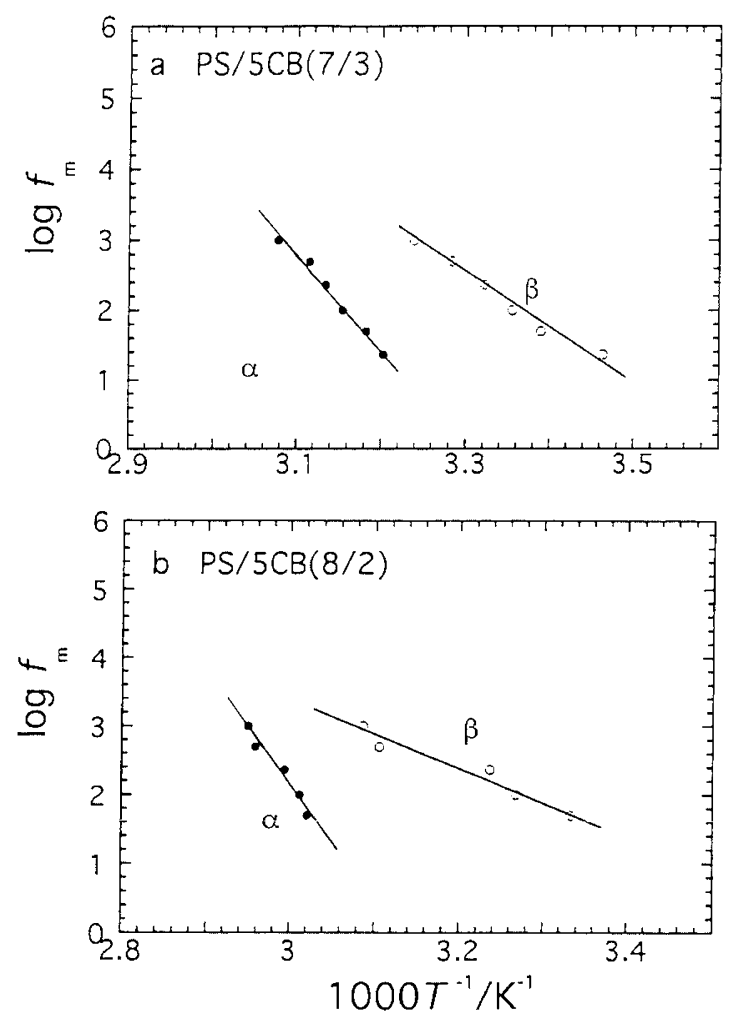

Figure 7. Arrhenius plots of the $\alpha$ and $\beta$ processes resolved with eq 1. a: $\mathrm{PS} / 5 \mathrm{CB}(7 / 3)$, b: $\operatorname{PS} / 5 \mathrm{CB}(8 / 2)$.

signment was also confirmed by measurements of the second moment of proton nuclear magnetic resonance $\left({ }^{1} \mathrm{H}\right.$ NMR) on solutions of PS dissolved in ordinary toluene $\left(\mathrm{C}_{8} \mathrm{H}_{8}\right)$ and fully deuterated toluene $\left(\mathrm{C}_{8} \mathrm{D}_{8}\right) .{ }^{4}$

Hereafter we distinguish the relaxation processes of the PS/5CB and those of the PS/toluene systems by using the codes 5CB and Tol, respectively. The $\alpha(5 \mathrm{CB})$ peak of $\mathrm{PS} / 5 \mathrm{CB}(7 / 3)$ measured at $100 \mathrm{~Hz}$ locates at $317 \mathrm{~K}$ as shown in Figure $6 \mathrm{~b}$. On the other hand $T_{\mathrm{g}}$ of this blend is $300 \mathrm{~K}$. Usually the primary $(\alpha)$ relaxation of an amorphous polymer at $100 \mathrm{~Hz}$ occurs about 10 to $20 \mathrm{~K}$ above the $T_{\mathrm{g}}$. Therefore it is reasonable to regard that the $\alpha(5 \mathrm{CB})$ peak corresponds to the $\alpha(\mathrm{Tol})$ peak and is due to segmental motions of PS. The $\beta(5 \mathrm{CB})$ peak locates slightly below $T_{\mathrm{g}}$ and corresponds to the 
Table II. Activation energy of the $\alpha$ and $\beta$ processes

\begin{tabular}{ccc}
\hline \multirow{2}{*}{ Blend } & $E_{\alpha}$ & $E_{\beta}$ \\
\cline { 2 - 3 } & $\mathrm{kJ} / \mathrm{mol}$ & $\mathrm{kJ} / \mathrm{mol}$ \\
\hline $\mathrm{PS} / 5 \mathrm{CB}(7 / 3)$ & 264 & 150 \\
$\mathrm{PS} / 5 \mathrm{CB}(8 / 2)$ & 320 & 96 \\
\hline
\end{tabular}

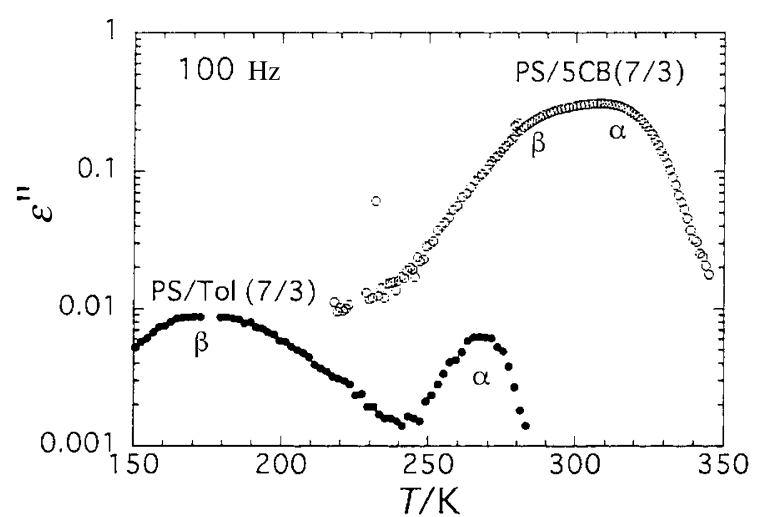

Figure 8. Comparison of the temperature dependencies of $\varepsilon^{\prime \prime}$ at $100 \mathrm{~Hz}$ between $\mathrm{PS} / 5 \mathrm{CB}(7 / 3)$ and $\mathrm{PS} /$ toluene(7/3).

$\beta$ (Tol) peak. Here it should be noted that the observed loss peaks in PS/5CB blends are totally due to motions of the $5 \mathrm{CB}$ molecules since the dipole moment of $5 \mathrm{CB}$ is much larger than that of PS. The PS chains govern the dynamics of the system but its dielectric response is too small to be observed. Thus both the $\alpha(5 \mathrm{CB})$ and $\beta(5 \mathrm{CB})$ processes reflect motions of the $5 \mathrm{CB}$ molecules. The above consideration leads us to consider that there are two kinds of $5 \mathrm{CB}$ molecules. A part of the 5CB molecules move cooperatively with the PS segments and cause the $\alpha(5 \mathrm{CB})$ process but the other move independently in the glassy PS matrix and cause the $\beta$ process. The $5 \mathrm{CB}$ molecules contributing to the $\alpha(5 \mathrm{CB})$ process are frozen-in below $T_{\mathrm{g}}$.

In Figure 7 it is seen that the $\alpha(5 \mathrm{CB})$ and $\beta(5 \mathrm{CB})$ processes tend to merge with increasing temperature. The relationship between this phenomenon and the dynamic heterogeneity in the temperature region above the $T_{\mathrm{g}}$ is an interesting future issue. Probably the size of the cooperative region decreases with increasing temperature and the degree of heterogeneity decreases.

\section{Dielectric Relaxation Strength}

In this section we will roughly assess the ratio of the 5CB molecules involved in the $\alpha$ and the $\beta$ processes by calculating the relaxation strengths $\Delta \varepsilon_{\alpha}$ for the $\alpha$ process and $\Delta \varepsilon_{\beta}$ for the $\beta$ process from the values of the parameters of eq 1 . Before doing so the total dielectric relaxation strength $\Delta \varepsilon\left(=\Delta \varepsilon_{\alpha}+\Delta \varepsilon_{\beta}\right)$ was determined from the Cole-Cole plots at temperatures where the loss peaks are in the midst of the frequency range used in this study. We have also determined $\Delta \varepsilon$ by curve fitting with the Havliriak-Negami equation. ${ }^{29}$
Table III. Total Relaxation Strength $\Delta \varepsilon=\Delta \varepsilon_{\alpha}+\Delta \varepsilon_{\beta}$

\begin{tabular}{ccc}
\hline Blend & $T$ & $\Delta \varepsilon$ \\
\hline $\mathrm{PS} / 5 \mathrm{CB}(7 / 3)$ & 323 & $2.60 \pm 0.3$ \\
$\mathrm{PS} / 5 \mathrm{CB}(8 / 2)$ & 330 & $1.25 \pm 0.2$ \\
\hline
\end{tabular}

The results are given in Table III. Due to the limited frequency range, the error was estimated and given in the same table. According to Onsager, $\Delta \varepsilon$ is given by ${ }^{30}$

$$
\Delta \varepsilon=\frac{4 \pi N \mu^{2}}{3 k_{\mathrm{B}} T} \frac{\left(\varepsilon_{\mathrm{U}}+2\right)^{2} \varepsilon_{\mathrm{R}}}{3\left(2 \varepsilon_{\mathrm{R}}+\varepsilon_{\mathrm{U}}\right)}
$$

where $N$ is the number of the monomeric unit in unit volume, $\mu$ the effective dipole moment, $\varepsilon_{U}$ the unrelaxed dielectric constant, and $\varepsilon_{\mathrm{R}}$ the relaxed dielectric constant. From the present experimental data, $\mu$ is determined to be $3.6 \pm 0.4 \mathrm{D}$. This value is close to the dipole moment of cyanobenzene $(4.0 \mathrm{D})^{31}$ and therefore we conclude that the $5 \mathrm{CB}$ molecules rotate without constraint in the matrix of PS.

Although it is desirable to determine $\Delta \varepsilon$ from the frequency dependence curves of $\varepsilon^{\prime}$ or $\varepsilon^{\prime \prime}$, it is difficult to resolve the loss curve shown in Figure 5 into the contributions of the $\alpha\left(\Delta \varepsilon_{\alpha}\right)$ and the $\beta\left(\Delta \varepsilon_{\beta}\right)$ processes. Therefore we roughly estimated the ratio of $\Delta \varepsilon_{\alpha}$ and $\Delta \varepsilon_{\beta}$ from the $T$-dependence curves of $\varepsilon^{\prime \prime}$. As indicated in the Appendix, $\Delta \varepsilon$ is approximately given by

$$
\Delta \varepsilon \cong \frac{2 E}{\pi R T_{\mathrm{m}}^{2}} \int_{0}^{\infty} \varepsilon^{\prime \prime} \mathrm{d} T
$$

where $E$ is the apparent activation energy, $R$ the gas constant, and $T_{\mathrm{m}}$ the loss maximum temperature. If the $T$-dependencies of $\varepsilon^{\prime \prime}$ for the $\alpha$ and $\beta$ processes conform to eq 1 , eq 3 can be calculated as shown in the Appendix and $\Delta \varepsilon$ of each process is given by

$$
\Delta \varepsilon_{\mathrm{j}} \cong \frac{E_{\mathrm{j}} K_{\mathrm{j}}}{R \gamma_{\mathrm{j}} T_{\mathrm{j}}^{2}}
$$

where $\mathrm{j}$ represents $\alpha$ or $\beta$. The results of calculation are listed in Table I. It is seen that the total relaxation strength $\Delta \varepsilon_{\alpha}+\Delta \varepsilon_{\beta}$ agrees approximately with that obtained from the $f$-dependence of $\varepsilon^{\prime \prime}$ with eq 2. Small discrepancy is ascribed to the approximation used in the eqs 1 and 3. The ratio $\phi_{\alpha}=\Delta \varepsilon_{\alpha} /\left(\Delta \varepsilon_{\alpha}+\Delta \varepsilon_{\beta}\right)$ can be regarded as the fraction of the $5 \mathrm{CB}$ molecules moving cooperatively with the PS segments. This value becomes 0.25 and 0.63 for $\mathrm{PS} / 5 \mathrm{CB}(7 / 3)$ and $\mathrm{PS} / 5 \mathrm{BC}(8 / 2)$, respectively, indicating that $\phi_{\alpha}$ increases with decreasing content of $5 \mathrm{CB}$. When the content of $5 \mathrm{CB}$ is low, most of the 5CB molecules are surrounded by the PS segments and move cooperatively with PS. With increasing $5 \mathrm{CB}$ content the probability that the $5 \mathrm{CB}$ molecules are surrounded by both the PS segments and the $5 \mathrm{CB}$ molecules increases and those molecules can move 

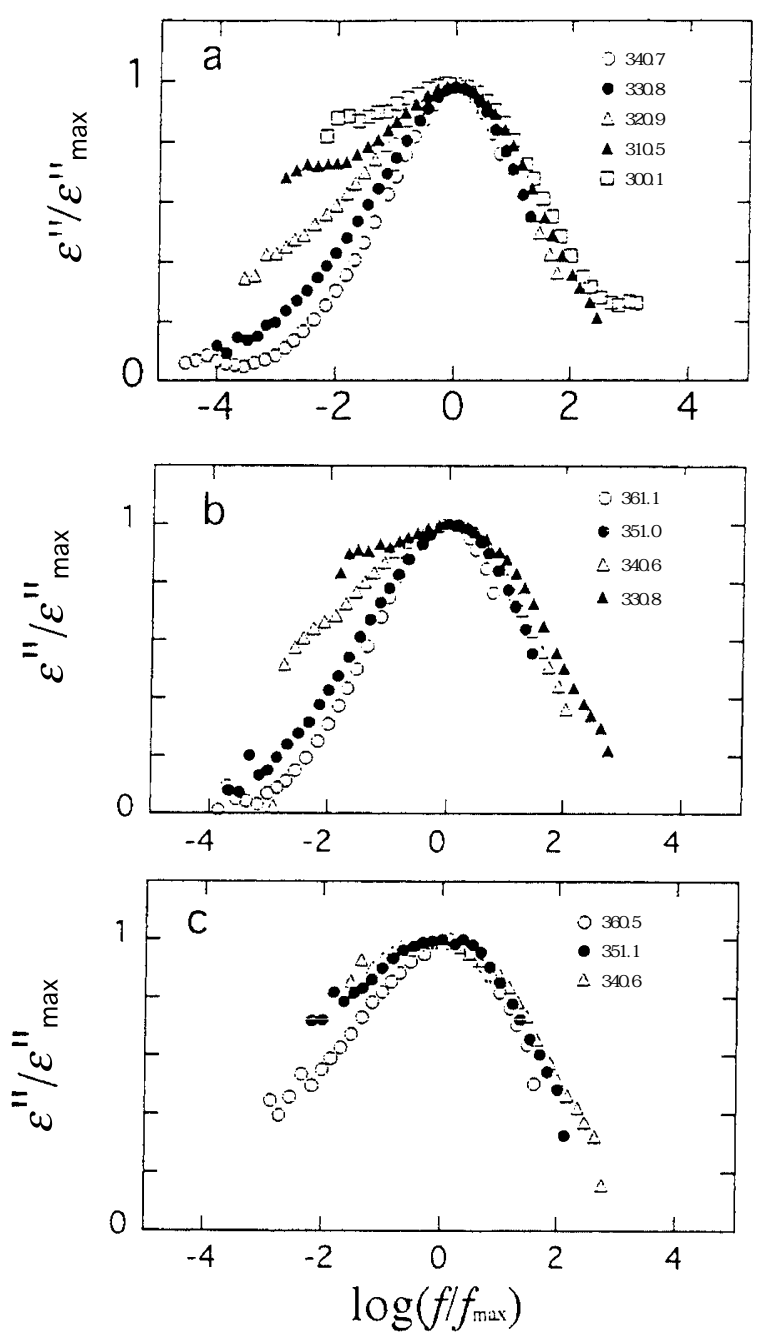

Figure 9. Normalized $\varepsilon^{\prime \prime}$ curves at various temperatures indicated in the figure. a: $\operatorname{PS} / 5 \mathrm{CB}(7 / 3), b$ : $\operatorname{PS} / 5 \mathrm{CB}(8 / 2)$, and $c$ : $\mathrm{PS} / 5 \mathrm{CB}(9 / 1)$.

without constraint of the PS chains. It is also expected that $\phi_{\alpha}$ decreases with increasing temperature. To examine this behavior, we need to determine the $\Delta \varepsilon$ for the $\alpha$ and $\beta$ processes at the same temperature. The data given in Table I are not sufficient for this purpose. The temperature dependence of $\phi_{\alpha}$ is remained as an intriguing issue.

The above results provide a piece of information on the average size of the units of segmental motions. The sizes of the molecules of $5 \mathrm{CB}$ and toluene along their long axes are 1.6 and $0.56 \mathrm{~nm}$, respectively. The toluene molecule is smaller than the size of the segments and can move independently of the PS segments. On the other hand the 5CB molecule is about three times larger than toluene and hence it moves cooperatively with the PS segments. The present result suggests that the average size of the PS segments is similar to the size of the $5 \mathrm{CB}$ molecules.

\section{Shape of Loss Curve and Spatial Heterogeneity}

For concentrated solutions of poly(vinyl acetate) in toluene, Yada et al. ${ }^{32}$ and Nakazawa et al. ${ }^{33}$ reported the broadening of the $\varepsilon^{\prime \prime}$ curves for the $\alpha$ relaxation process. The width of the $\varepsilon^{\prime \prime}$ peaks $(\alpha)$ broadened with decreasing temperature in proportion to $1 /(T-$ $\left.T_{\mathrm{o}}\right)^{2}$ where $T_{\mathrm{o}}$ denotes the critical temperature of the Vogel-Fulcher equation. ${ }^{27,28}$ This behavior indicates that there exists spatial distribution of the local friction for the segmental motion of poly(vinyl acetate) and has been explained by assuming a Gaussian distribution of local concentration. ${ }^{34}$ Those works were extended by Yoshizaki et al. to the PS/alkylbenzene system. ${ }^{1}$ They indicated that the amplitude of the concentration fluctuation depends on the quality of the solvent and with the deterioration of the solvent quality, the broadening of the loss curve is enhanced. Figures $9 a, 9 b$, and $9 c$ show the normalized $\varepsilon^{\prime \prime}$ curves for $\mathrm{PS} / 5 \mathrm{CB}(7 / 3),(8 / 2)$, and $(9 / 1)$, respectively. It is seen that the normalized $\varepsilon^{\prime \prime}$ curves broaden with decreasing temperature as reported previously. ${ }^{1,32,33}$

Since the dielectric measurements were carried out around the upper critical solution temperature (see Figure 2), $5 \mathrm{CB}$ is a poor solvent for PS. From the results reported by Yoshizaki et al., ${ }^{1}$ we expect that the present system exhibits relatively strong broadening of the $\varepsilon^{\prime}$ curves with decreasing temperature. In Figure 9, the broadening seen in the low frequency side is responsible to the merging process of the $\alpha$ and $\beta$ processes as discussed above. The broadening seen in the high frequency side is responsible to the broadening of the $\beta$ peak and reflects concentration fluctuation. Similar broadening behavior of the $\beta$ process was also observed for the PS/butylbenzene system. ${ }^{1}$ However we see that the extent of the broadening in both the PS/5CB and $\mathrm{PS} /$ butylbenzene systems is relatively small compared with the $\alpha$ process. In order to assess the amplitude of concentration fluctuation, we need the concentration dependence of the relaxation time for the $\beta$ process but such data are not sufficient in the present study.

\section{CONCLUSION}

In an effort to elucidate the dynamics of low molecular weight molecules dissolved in polymer matrices, we have investigated the dielectric relaxation in blends of polystyrene PS and 4- $n$-pentyl-4' -cyanobiphenyl (5CB) having the size $c a$. three times larger than the toluene molecule. The temperature $(T)$ dependence of the dielectric loss factor $\varepsilon^{\prime \prime}$ exhibits broad bimodal $\varepsilon^{\prime \prime}$ peak consisting of the $\alpha$ and $\beta$ rocesses. In comparison with the dielectric behavior of the PS/alkylbenzene systems, the $\alpha$ process are assigned to the cooperative motion 
of the PS segments and the $5 \mathrm{CB}$ molecules and the $\beta$ process to independent rotation of the part of the $5 \mathrm{CB}$ molecules, respectively. From the relaxation strengths for the $\alpha$ and $\beta$ processes, the fraction of the 5CB molecules moving cooperatively with PS has been estimated to be 0.25 and 0.63 for the blends containing 30 and $20 \mathrm{wt} \%$ of $5 \mathrm{CB}$, respectively.

\section{APPENDIX}

The relaxation strength $\Delta \varepsilon$ is given by

$$
\Delta \varepsilon=\frac{2}{\pi} \int_{-\infty}^{\infty} \varepsilon^{\prime \prime} \mathrm{d} \ln \omega
$$

where $\omega$ denotes the angular frequency. Since $\varepsilon^{\prime \prime}$ is given by the sum of the Debye functions, $\ln \omega$ can be replaced by $\ln \tau$ where $\tau$ is the relaxation time. In the relatively narrow temperature range $\ln \tau$ is linear to $E / R T$ and eq $\mathrm{A} 1$ is approximately rewritten as

$$
\Delta \varepsilon \cong \frac{2 E}{\pi R} \int_{0}^{\infty} \frac{\varepsilon^{\prime \prime}}{T^{2}} \mathrm{~d} T
$$

where $E$ is the apparent activation energy. Since the integration around a relatively narrow region of the loss maximum temperature $T_{\mathrm{m}}$ is important, we may approximate that $T$ is a constant equal to $T_{\mathrm{m}}$. Then we obtain eq 3 . When $\varepsilon^{\prime \prime}$ is given by eq 1 , the integral of eq 3 is equal to the integration of $\operatorname{sech}(\gamma x) / 2$ over the range from $-\infty$ to $\infty$ where $x$ is $T-T_{\mathrm{m}}$. Since the integral is equal to $\pi / 2 \gamma$, we obtain eq 4 .

\section{REFERENCES}

1. K. Yoshizaki, O. Urakawa, and K. Adachi, Macromolecules, 36, 2349 (2003).

2. A. K. Rizos, L. Petihakis, K. L. Ngai, J. H. Wu, and A. F. Yee, Macromolecules, 32, 7921 (1999).

3. M. Mashimo, J. Chem. Phys., 67, 2651 (1977).

4. K. Adachi, I. Fujihara, and Y. Ishida, J. Polym. Sci., Polym. Phys. Ed., 13, 2155 (1975).

5. Th. Blochowicz, C. Karle, A. Kudlik, P. Medicj, I. Roggatz, M. Vogel, Ch. Tschirwitz, J. Wolber, J. Senker, and E. Rossler, J. Phys. Chem. B, 103, 4032 (1999).

6. A. K. Rizos, R. M. Johnsen, W. Brown, and K. L. Ngai, Macromolecules, 28, 5450 (1995).
7. R. Casalini, P. G. Santangelo, and C. M. Roland, J. Chem. Phys., 117, 4585 (2002).

8. B. J. Cauley, C. Cipriani, K. Ellis, A. K. Roy, A. A. Jones, P. T. Inglefield, B. J. McKinley, and R. P. Kambour, Macromolecules, 24, 403 (1991).

9. G. C. Chung, J. A. Kornfield, and S. D. Smith, Macromolecules, 27, 964 (1994).

10. A. A. Jones, P. T. Inglefield, Y. Liu, A. K. Roy, and B. J. Cauley, J. Non-Cryst. Solids, 131, 556 (1991).

11. C. T. Thurau and M. D. Ediger, J. Chem. Phys., 118, 1996 (2003).

12. J. Pilar and J. Labsky, Macromolecules, 36, 913 (2003).

13. P. Bergquist, Y. Zhu, A. A. Jones, and P. T. Inglefield, Macromolecules, 32, 7925 (1999).

14. C. J. Montrose and T. A. Litovitz, J. Acoust. Soc. Am., 47, 1250 (1970).

15. G. Adam and J. H. Gibbs, J. Chem. Phys., 43, 139 (1965).

16. K. L. Ngai, R. W. Rendel, A. K. Rajagopal, and S. Teiler, Ann. N. Y. Acad. Sci., 484, 150 (1986).

17. S. Matsuoka and X. Quan, Macromolecules, 24, 2770 (1991).

18. K. Adachi, Macromolecules, 23, 1816 (1990).

19. A. Alegria, J. Colmenerro, K. L. Ngai, and C. M. Roland, Macromolecules, 27, 4486 (1994).

20. F. Alvarez, A. Alegria, and J. Colmenerro, Macromolecules, 30, 597 (1997).

21. E. Donth, J. Non Cryst. Solids, 53, 325 (1982).

22. W. Ahn, C. Y. Kim, H. Kim, and C. S. Kim, Macromolecules, 25, 5002 (1992).

23. Z. Lin, H. Zhang, and Y. Yang, Phys. Rev. E, 58, 5867 (1998).

24. J. Zhu, G. Xu, J. Ding, and Y. Yang, Macromol. Theory Simul., 8, 409 (1999).

25. T. Bouchaour, F. Benmouna, F. Roussel, J.-M. Buisine, X. Coqueret, M. Benmouna, and U. Maschke, Polymer, 42, 1663 (2001).

26. N. G. McCrum, B. E. Read, and G. Williams, "Anelastic and Dielectric Effects in Polymeric Solids," John Wiley \& Sons Inc., New York, N.Y., 1967.

27. H. Vogel, Phys. Z., 22, 645 (1921).

28. G. A. Fulcher, J. Am. Ceram. Soc., 8, 339 (1925).

29. S. Havriliak and S. Negami, J. Polym. Sci., C, 14, 99 (1966).

30. L. Onsager, J. Am. Chem. Soc., 58, 1486 (1936).

31. C. P. Smyth, "Dielectric Behavior and Structure," MacGrawHill, New York, N.Y., 1955, Chapt. 10.

32. M. Yada, M. Nakazawa, O. Urakawa, Y. Morishima, and K. Adachi, Macromolecules, 33, 3368 (2000).

33. M. Nakazawa, O. Urakawa, and K. Adachi, Macromolecules, 33, 7898 (2000).

34. A. Zetsche and E. W. Fischer, Acta Polym., 45, 168 (1994). 